\title{
Characterisation of parallel kinematic machines based on structural workspaces
}

\author{
Javier Corral ${ }^{1}$, Charles Pinto ${ }^{1, a}$, Oscar Altuzarra ${ }^{1}$ and Asier Zubizarreta ${ }^{2}$ \\ 1 Department of Mechanical Engineering, University of the Basque Country (UPV/EHU), 48013 Bilbao, Spain \\ 2 Automatics and System Engineering Department, University of the Basque Country (UPV/EHU), 48013 Bilbao, Spain
}

Received 8 June 2012, Accepted 1 February 2013

\begin{abstract}
In this paper, we present the static and dynamic structural characterisation of a low-mobility parallel kinematic manipulator, involving analysis of its stiffness and vibrational dynamic behaviour. The study starts by building numerical models of the behaviour of the manipulator to be compared to experimental measurements from a prototype. For the case study, we consider a four-degree-of-freedom $\left(x, y, z, \theta_{z}\right)$ manipulator with prismatic actuators designed by the COMPMECH research group at the University of the Basque Country. The characterisation allows the behaviour of the static and dynamic stiffness, as well as the natural frequencies of the manipulator, to be mapped in the manipulator workspace. These maps together with kinematic, static and dynamic constraints lead to the definition of operational, static, dynamic and structural workspaces, respectively. Further, we analyse the modes of the manipulator to determine dynamic displacements, these being key in the performance in the machining tasks for which the robot was designed.
\end{abstract}

Key words: Parallel manipulator / stiffness / natural frequencies / mode shapes / vibrations / workspace

\section{Introduction}

Improvement in industrial production indicators for high added value parts, such as those for the aviation, aerospace and automotive sectors, is limited by the high quality standards required by these sectors. Accordingly, to increase productivity manufacturing processes need to be developed simultaneously with technical capacities, and task automation using robotic manipulators plays a key role in this. Most industrial applications require robots with high accuracy, high stiffness and dynamic characteristics that ensure vibrational phenomena do not interfere with the performance of the tasks for which the robots have been designed. In this paper, we present a study of the static and dynamic structural characteristics of a four-degree-of-freedom parallel manipulator for machining applications in the aviation industry. Using a previously validated model, we obtain maps which represent the behaviour of the manipulator in its workspace clearly and intuitively. Knowing the structural requirements for a given application, for instance, the minimum stiffness and range of natural frequencies for operation of the system, it is possible to delimit the aforementioned maps and thereby obtain the workspaces associated with

a Corresponding author: charles.pinto@ehu.es these requirements. In this way, an operational region is defined within the workspace on the basis of the requirements for the specific application. Once this is achieved, we define workspaces in relation to the kinematic, static and dynamic constraints. To illustrate the usefulness of this approach, we characterise the structural properties of the ARABA I manipulator.

In the literature many design approaches for 3T1R manipulators can be found. Attending different criteria like kinematics or rigid solid dynamics even optimization procedures are carried out. In [1] the obtained design is the basis of a commercial product. Another design approach described the cancellation of the loads of gravity on the actuators validated by experimental tests carried out on the prototype. Also performance indexes variations are taken into account to determine the largest regular dextrous workspace enclosed in the Cartesian workspace [2] to ensure milling tool paths availability. In [3] it was shown that the input torques of the actuators with and without a payload of $20 \mathrm{~kg}$ are similar. A lot of studies take into account the stiffness of parallel manipulators in the design stage like in [4] where a theoretical method is proposed to derivate the stiffness.

Dynamic stiffness is a key factor in determining whether a machine operates correctly $[5,6]$. Indeed, all the dynamic structural properties of the manipulator should 

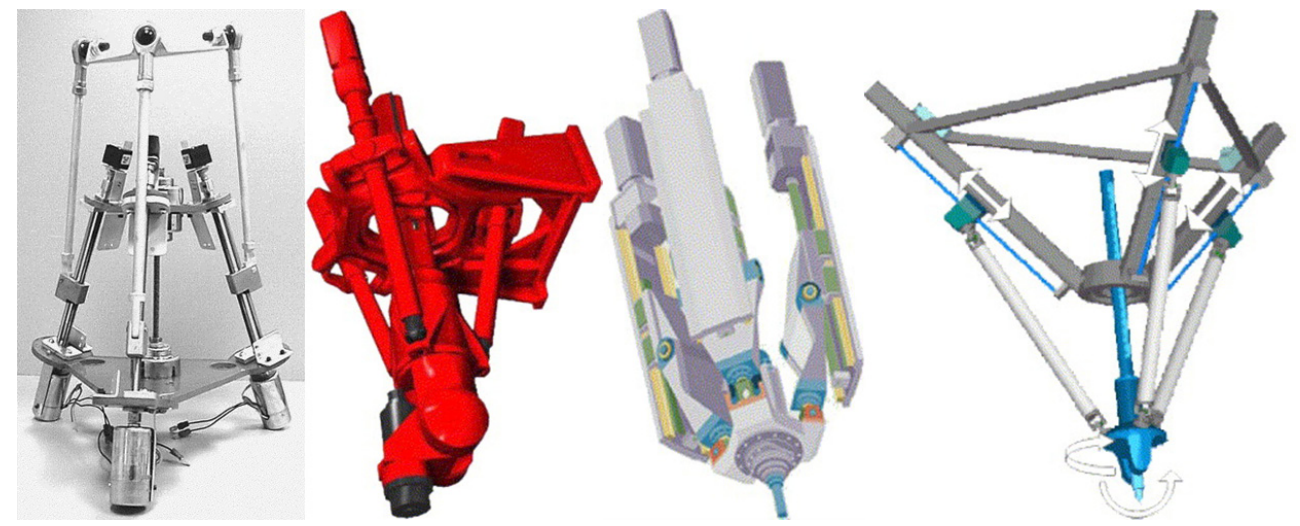

Fig. 1. Tripod-based machines: generic, Tricept, $Z^{3}$ Head and Georg V.

be considered. If not, dynamic excitation, such as that from actuators or tools mounted on the end-effector, may give rise to mechanical resonance in the system $[7,8]$. To avoid this undesirable phenomenon, the final user of the machine needs a simple, practical tool. This tool should enable suitability of a machine to be evaluated in terms of operational parameters of the process (cut depth, feed rate, cut speed, etc.) for which it has been designed and the operating conditions (workspace, velocities, accelerations, load capacity and power of the actuators, among others). To assess the maximum displacements of the end-effector an exhaustive analysis of the static stiffness should be carried out [9]. Otherwise, the objectives of the process may not be met: in terms of machining, the surface finish may not be sufficiently high quality, and in pick and place tasks, positioning of parts may be inaccurate [10].

In relation to machining applications, several authors have studied the influence of actuator arrangement in manipulators for applications in the machine tool sector. For example, in [11], the authors compare tripod-based machines such as those shown in Figure 1.

Their analysis is based on configurations with extendable legs which give larger workspaces, while to increase the stiffness of the system it is better to use fixed-length legs with vertical actuators. In [12] a kinetostatic model of a tripod manipulator is used to demonstrate that the bending mode is most sensitive to stiffness while the maximum stiffness is obtained when operating the system vertically, the direction in which most of the components of the manipulator are principally under axial loading.

It is well known that the stiffness of parallel manipulators depends on their configuration [13] as well as the position and orientation of the end-effector, and it can be expected that these factors also affect the vibrational properties of the system. Accordingly, end-users need a tool that can analyse the vibrational behaviour of manipulators in their workspace. Notably, there are certain tasks, such as surface polishing, that do not require particularly high actuator forces but in which tool rotation may generate vibrations, the effect of which should not be underestimated [14]. Polishing is a finishing process to smooth the surface of machined parts using an abra- sive fixed to a work wheel. The wheel is usually driven by a rotating motor, a source of dynamic excitation, that may cause undesirable vibrations and even resonance problems.

Other authors have already proposed particular subregions for a given workspace. For example, [15] defines a mechanical workspace as a region of the workspace where machined position defects due to machine tool structure are within tolerances. Later on [16], went into details for a particular machine tool for drilling. This result also leaded to derivate some intuitive results about stiffness behaviour of the parallel machine.

In the next section, a case study for a particular parallel manipulator is presented. Then, in Section 3 static stiffness of the manipulator is obtained and, after that, in Section 4 the dynamic stiffness as well. From these studies several workspaces are proposed to finally obtain the structural workspace. In the last section, the most outstanding conclusions are revealed.

\section{Case study: the ARABA I manipulator}

The ARABA I parallel manipulator is a four-degreeof-freedom (DOF) low-mobility device (Fig. 2). Here we outline its key characteristics, those essential for understanding its static behaviour, as it has been described in detail in a previous publication [17]. The purpose of this manipulator is to help with machining tasks and, accordingly, it is required to have the precision and stiffness necessary for such processes. Despite the fact that, in principle, only the three translational DOFs are needed for this type of application, the addition of a fourth $D O F$, rotation in a plane perpendicular to the end-effector, means that it can machine uneven surfaces.

The architecture of ARABA I is based around a fixed platform and a mobile platform or end-effector. The guides, the fixed component, are not perfectly aligned, rather they are offset by a distance $a$. The two platforms are connected by four identical legs composed of several components. The basic kinematic chain of each leg is PRPaRR; that is, each leg is connected to the guides through an actuated prismatic pair, then there is a rotating pair $\left(B_{i}\right)$, an articulated parallelogram pair and, 


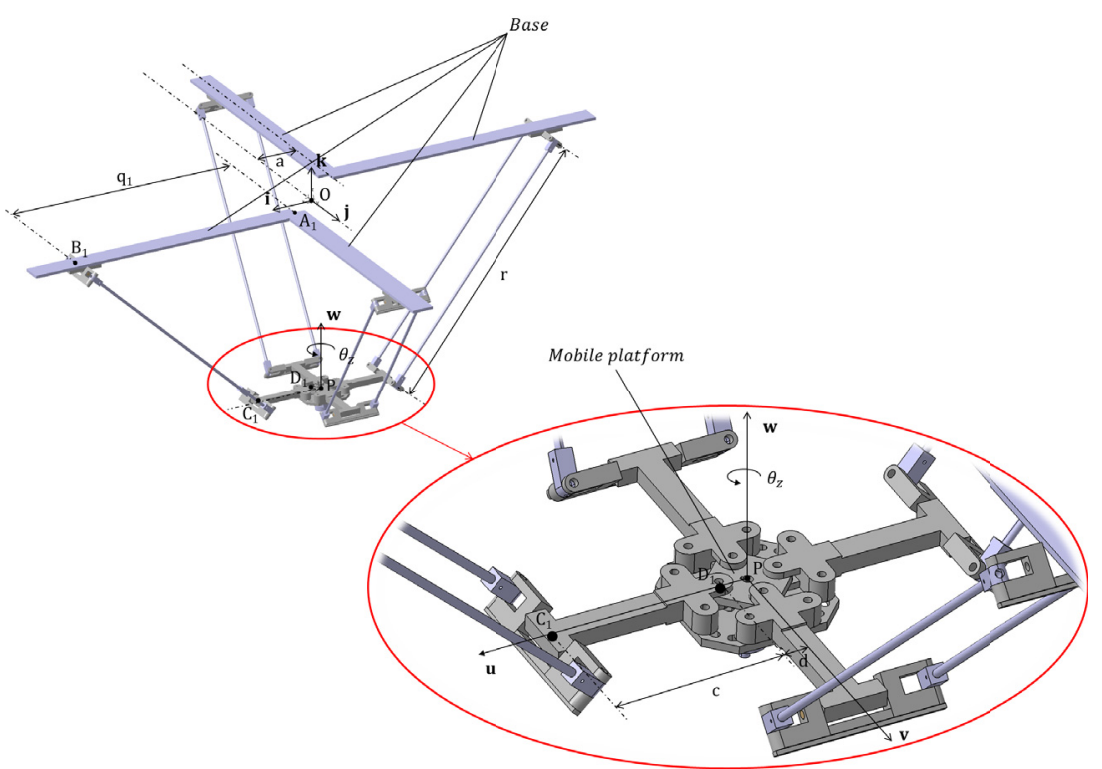

Fig. 2. Kinematic skeleton of the ARABA I manipulator.

finally, there are two further pairs that rotate about mutually perpendicular axes, $C_{i}$ and $D_{i}$ respectively. The key geometric parameters are: lengths, $b$ and $r$, of the short and long rods of the articulated parallelogram; the length, $c$, of the component $C_{i} D_{i}$ connecting the legs to the mobile platform; and the length, $d$, of the half-diagonal of the mobile platform itself (a regular rhombus).

Having outlined the architecture of the ARABA I, we should comment on the number and nature of the degrees of freedom of this manipulator. As indicated above, the mobile platform has four DOFs, the three translational movements along the Cartesian axes and one rotational around an axis perpendicular to the plane of the mobile platform $(3 T 1 R)$. Analysis of these DOFs applying any of the classical criteria fails to produce a satisfactory solution as there are certain movements in parallel directions among the rotational and translational pairs. In this case, it is easier to obtain an expression to describe the motion pattern of the system using Group Theory as detailed in [17].

\subsection{Kinematic model}

\subsubsection{Direct kinematic problem}

The objective of the direct kinematic problem is to obtain the value of the output parameters, defined with respect to the position vector $P\left(x_{p}, y_{p}, z_{p}\right)$ of the centre of the mobile platform and its angular position about the $Z$ axis (given by $\theta_{z}$ ) on the basis of the values of the input variables of the manipulator, that is, the lengths of the linear actuators, $q_{1}, q_{2}, q_{3}$ and $q_{4}$. For this, the position of the point $P$ is expressed in terms of each of the kinematic chains of the manipulator (Fig. 3) as:

$\boldsymbol{p}=\boldsymbol{a}_{i}+\left(\boldsymbol{b}_{i}-\boldsymbol{a}_{i}\right)+\left(\boldsymbol{c}_{i}-\boldsymbol{b}_{i}\right)+\left(\boldsymbol{d}_{i}-\boldsymbol{c}_{i}\right)+\left(\boldsymbol{p}_{i}-\boldsymbol{d}_{i}\right) i=1 \ldots 4$

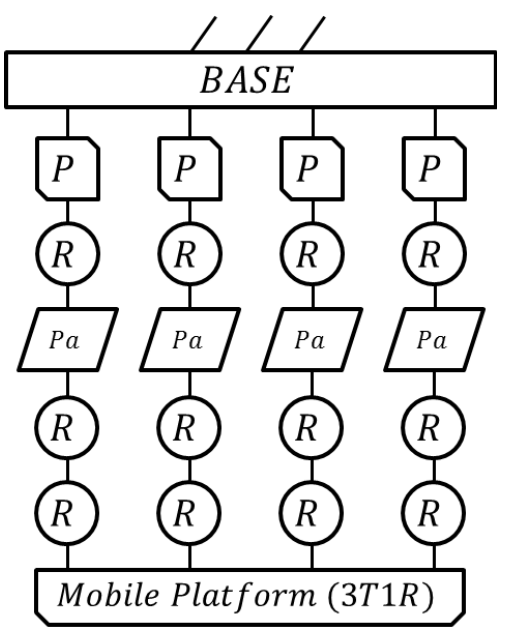

Fig. 3. Diagram of the linkage in the ARABA I manipulator.

where $\boldsymbol{x}_{i}$ is the position vector of point $x_{i}$. Considering geometric and kinematic constraints and applying various mathematical transformations (see [17]), the following set of non-linear equations describing the position of the mobile platform can be obtained:

$$
\begin{aligned}
& \left(x_{P}+d \cos \theta_{z}-n_{1}\right)^{2}+\left(y_{P}+d \sin \theta_{z}-a\right)^{2}+z_{P}^{2}=r^{2} \\
& \left(x_{P}-d \sin \theta_{z}-a\right)^{2}+\left(y_{P}+d \cos \theta_{z}-n_{2}\right)^{2}+z_{P}^{2}=r^{2} \\
& \left(x_{P}-d \cos \theta_{z}-n_{3}\right)^{2}+\left(y_{P}-d \sin \theta_{z}+a\right)^{2}+z_{P}^{2}=r^{2} \\
& \left(x_{P}+d \sin \theta_{z}+a\right)^{2}+\left(y_{P}-d \cos \theta_{z}+n_{4}\right)^{2}+z_{P}^{2}=r^{2}
\end{aligned}
$$

where

$$
n_{i}=q_{i}+a-c \quad i=1 \ldots 4
$$

From Equations (2), three independent linear equations can be found relating variables $x_{P}$ and $y_{P}$. Rearranging 


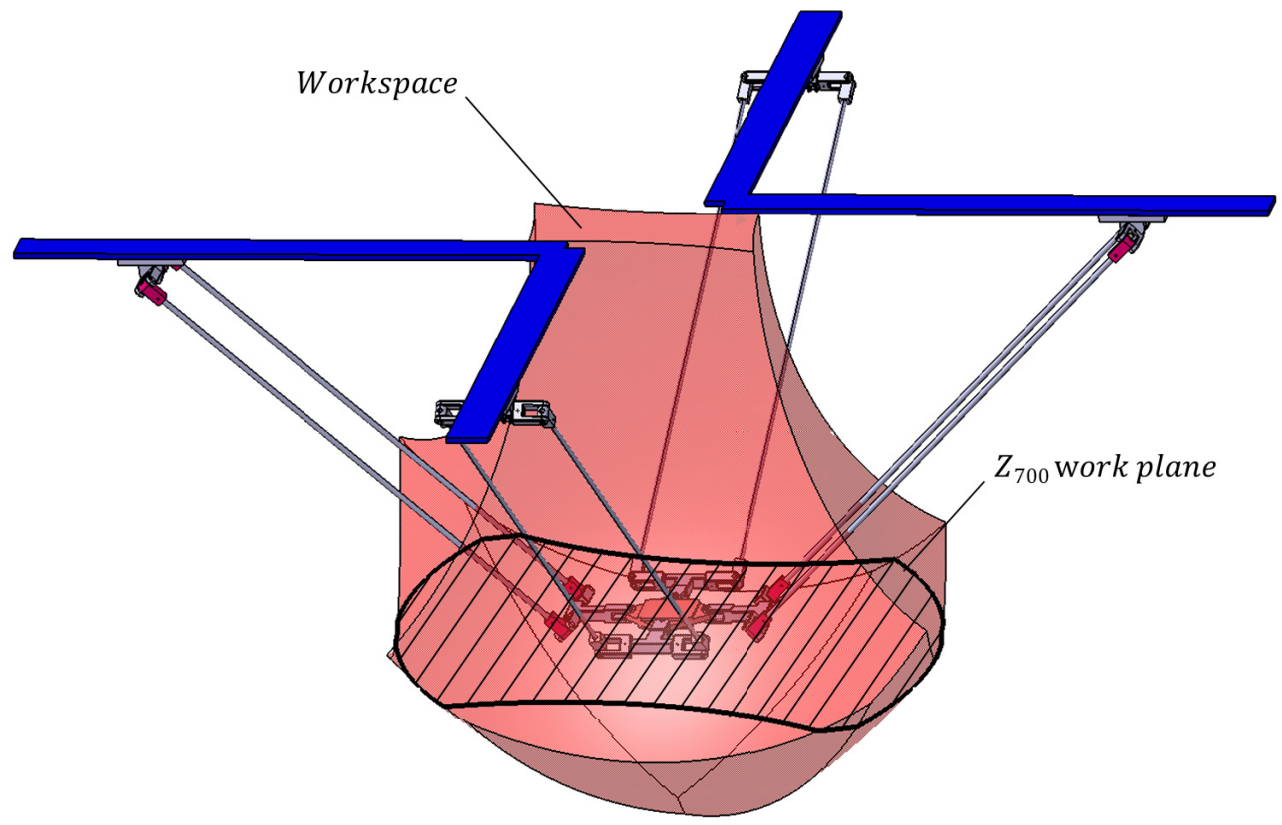

Fig. 4. Operational workspace and work plane of the manipulator.

these equations and making a change of variable, it is possible to obtain a fourth-order polynomial of the form:

$$
T_{4} s^{4}+T_{3} s^{3}+T_{2} s^{2}+T_{1} s+T_{0}=0
$$

Finding the real solutions of this polynomial, undoing the changes of variable and substituting back into Equations (2) yields values of $x_{P}, y_{P}, z_{P}$ and $\theta_{z}$ for the output variables.

\subsubsection{Inverse kinematic problem}

The objective of the inverse kinematic problem is to obtain values for the input variables, $q_{1}, q_{2}, q_{3}$ and $q_{4}$, as a function of the known outputs, that is, the position vector $\boldsymbol{p}$ and angle $\theta_{z}$ describing the position of point $P$ of the manipulator. The inputs $q_{i}$ can be calculated directly from Equations (2) and (3):

$$
\begin{array}{r}
q_{1}=x_{P}+d \cos \theta_{z}-a+c \\
\pm \sqrt{r^{2}-\left(y_{P}+d \sin \theta_{z}-a\right)^{2}-z_{P}^{2}} \\
q_{2}=y_{P}+d \cos \theta_{z}-a+c \\
\pm \sqrt{r^{2}-\left(x_{P}-d \sin \theta_{z}-a\right)^{2}-z_{P}^{2}} \\
q_{3}=-x_{P}+d \cos \theta_{z}-a+c \\
\pm \sqrt{r^{2}-\left(y_{P}-d \sin \theta_{z}+a\right)^{2}-z_{P}^{2}} \\
q_{4}=-y_{P}+d \cos \theta_{z}-a+c \\
\pm \sqrt{r^{2}-\left(x_{P}+d \sin \theta_{z}+a\right)^{2}-z_{P}^{2}}
\end{array}
$$

These equations yield $2^{4}$ solutions to the inverse kinematic problem. In order that these equations are satisfied, however, the sum of the discriminants should be greater than or equal to zero and this limits the number of possible solutions. Lastly, physical constraints on the actuator inputs must also be considered, further limiting the solutions and, in turn, the workspace.

\subsubsection{Workspace}

Having solved the kinematic problem and using the equations obtained, it is possible to identify the workspace of the manipulator. The volume shown in Figure 4 is for a constant angular position $\theta_{z}=0$ and with actuator stroke limits given by:

$$
\begin{aligned}
q_{i_{\min }} & =0.24 \mathrm{~m} \\
q_{i_{\max }} & =1 \mathrm{~m}
\end{aligned}
$$

This workspace can also be obtained as the intersection of the volumes reached by each of the legs of the manipulator, considering the characteristic lengths of each of the components using a simplified version of the method to geometrically compute the constant orientation workspace presented in [18]. Given that the manipulator has four DOFs, variations in stiffness as a function of its position and orientation can be explored by fixing, at least, two of the four position parameters, and creating a 3-D plot of the variations. Specifically, we consider a constant orientation angle, $\theta_{z}=0$ and, to fix a second parameter, planes of constant height. We then generate sets of points uniformly distributed across each plane, selecting a density as a function of the size of the work plane (at each height). Figure 4 illustrates the intersection of a constant plane $z=c t$ and the volume corresponding to the maximum workspace for which the experimental measurements were conducted. Specifically, this corresponds 


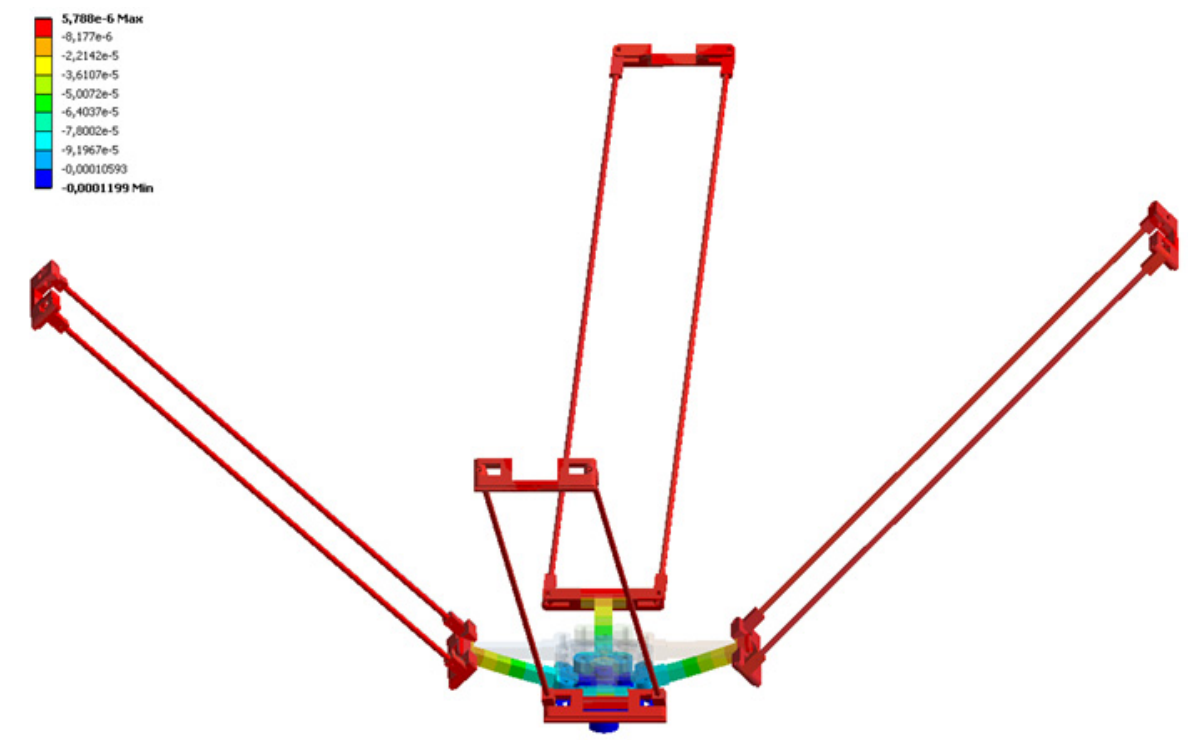

Fig. 5. Post-processing values for vertical displacement, $\delta_{z z}(\mathrm{~mm})$.

to the plane at a level of $z=-0.7 \mathrm{~m}$, hereon referred to as the $Z_{700}$ work plane.

In this work plane, calculations have been made for 25 points uniformly distributed across a rectangular region of $800 \times 600 \mathrm{~mm}$. Hereon, the region of workspace analysed, which is singularity free, is referred to as the operational workspace, $W_{\mathrm{op}}$. The manipulator has been positioned with the fixed platform at a higher level than the mobile one, as this is the most appropriate configuration in the applications for which the prototype was designed.

\section{Statics}

The static characterisation is based on analysis of the static stiffness. A finite element model was built, starting from the computer-aided design $(C A D)$ model used for the development of the prototype. In this case, given that the manipulator is composed of a large number of components, it was necessary to carefully select a mesh size to achieve a reasonable balance between computational cost and accuracy. For this, we opted for second-order hexahedral elements, giving a mesh composed of 65000 elements and 70 kinematic relations. A validation of the finite element model for the static analysis of stiffness has been published previously [9] and, therefore, here we directly present the values obtained for the points analysed. Figure 5 illustrates, for one of the positions considered, the post-processing results for vertical displacements, caused by a vertical load applied in the centre of the mobile platform. It can be seen that the largest displacements occur in the components connected to the mobile platform and in the platform itself.

The finite-element analysis yields values for the variation in stiffness which can be visualised using 3-D maps, as illustrated in Figure 6 for the $Z_{700}$ plane. Specifically, post-processed values for the displacement of the mobile platform, obtained from the numerical model, enable the components of stiffness in each direction to be identified. To consider the overall behaviour of the manipulator in its workspace, we plotted maps of the stiffness for a constant height (Fig. 6). Analysis of these maps reveals that the value of $k_{z z}$ component is greatest in the centre of the workspace and reduces as the mobile platform moves away from this point. Indeed, the maximum stiffness is found for $k_{z z}$ (over $4 \times 10^{5} \mathrm{~N} \cdot \mathrm{mm}^{-1}$ ). On the other hand, the $k_{x x}$ and $k_{y y}$ components have lower stiffness at the central point of the region studied (values of $10^{4} \mathrm{~N} . \mathrm{mm}^{-1}$ ) and, in contrast to $k_{z z}$, the stiffness in these directions does not vary so sharply when the mobile platform moves away from the central point.

\subsection{Static workspace}

Adding constraints on some elements of the rigidity matrix, the static workspace is given by:

$$
W_{K}=\left\{W_{\mathrm{op}} \backslash k_{i j_{\min }}<k_{i j}<k_{i j_{\max }}\right\}
$$

As an example, the static workspace associated with adding constraints on the $k_{z z}$ component is shown in Figure 7. We note that limiting the stiffness, the workspace obtained, $W_{K}$, is a subregion of the operational workspace, $W_{\mathrm{op}}$.

\section{Dynamics}

Using the same finite element model as for the static analysis, we obtain the natural frequencies and display the associated deformation modes graphically. Figure 8 presents the shapes of the first seven deformation modes. 

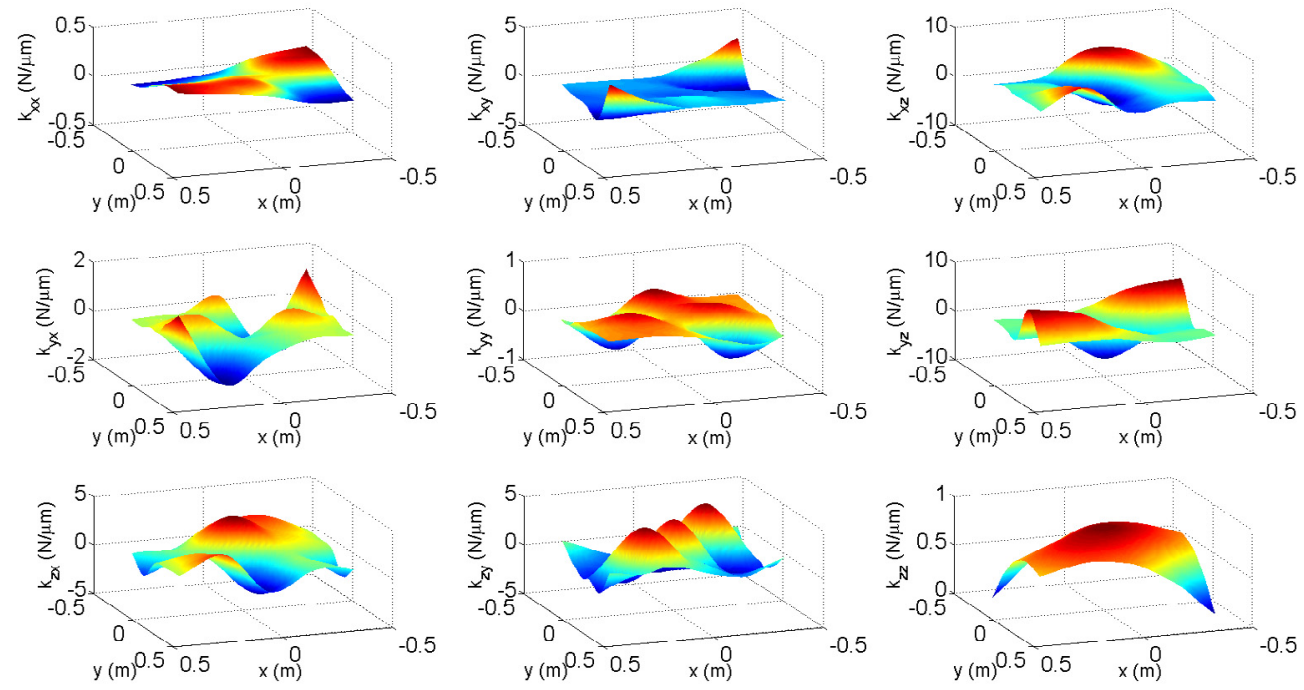

Fig. 6. Maps of rigidity $k_{i j}$ in the $Z_{700}$ plane.

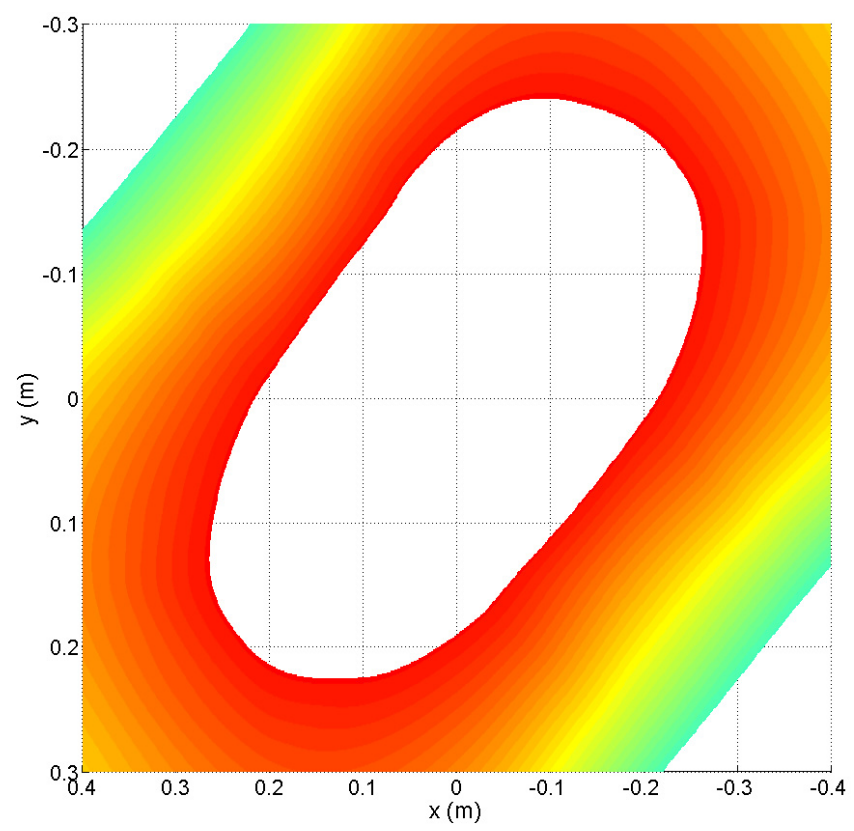

Fig. 7. Static workspace, $W_{K}$.

Between these first seven modes, various types of deformation occur. Essentially, the deformation corresponds to bending and torsional modes, the observed effect varying between modes. In almost all cases, the dominant effect is bending of the rods that form the leg quadrilaterals, given their narrowness. On the other hand, in some cases the mobile platform is displaced a considerable distance, while in others there is no shift. For a given application, it would be important to know the mode excited and the corresponding deformation of the manipulator, in order to assess whether there were negative consequences in terms of performance.

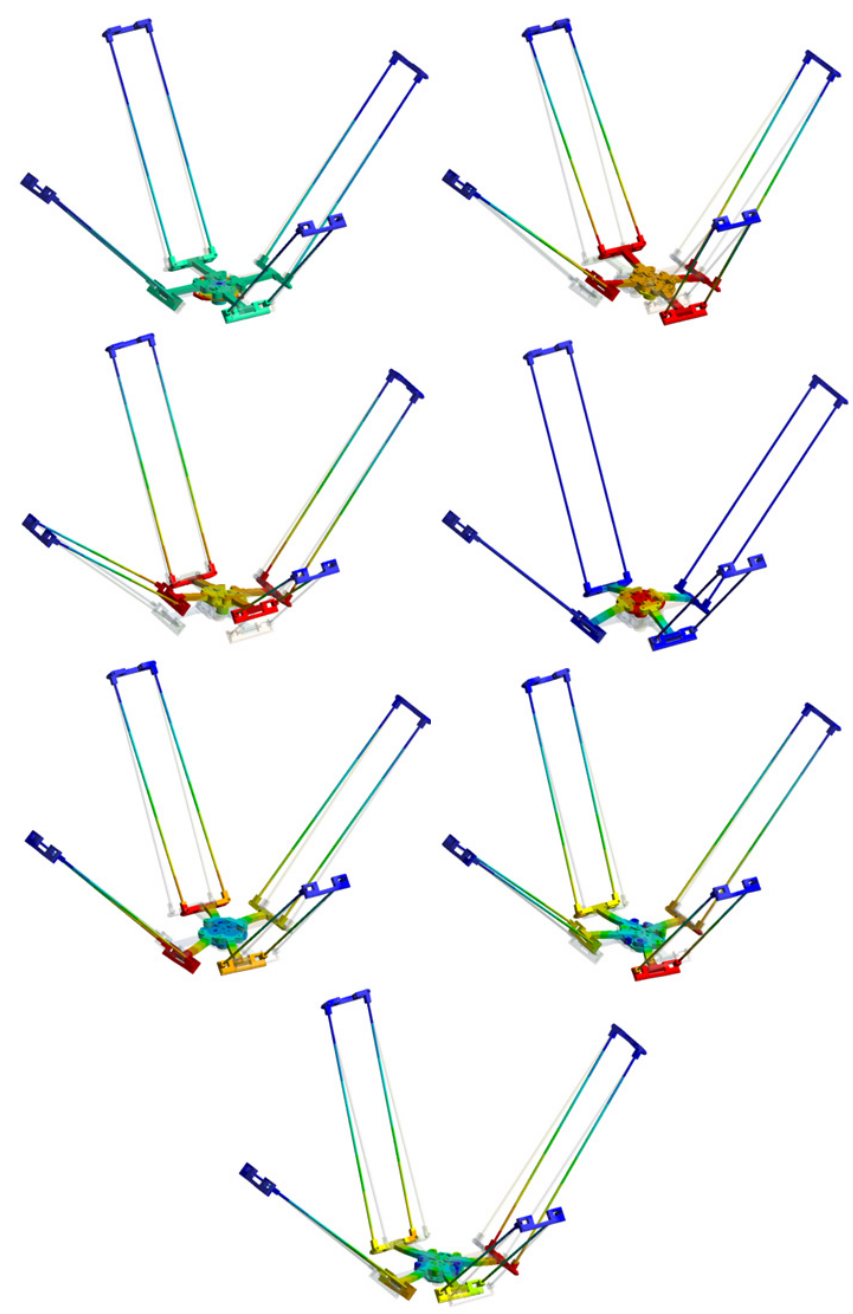

Fig. 8. M1 to M7 ARABA I mode shapes. 

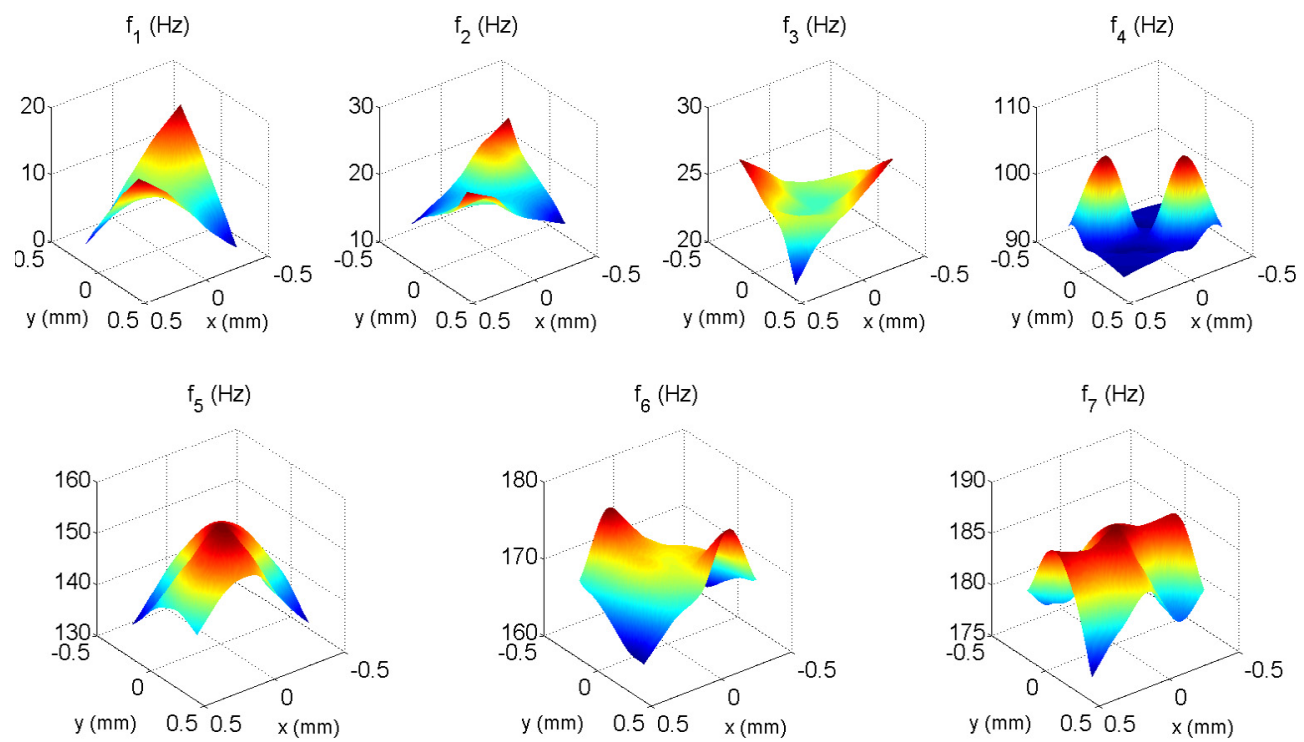

Fig. 9. ARABA I natural frequencies.

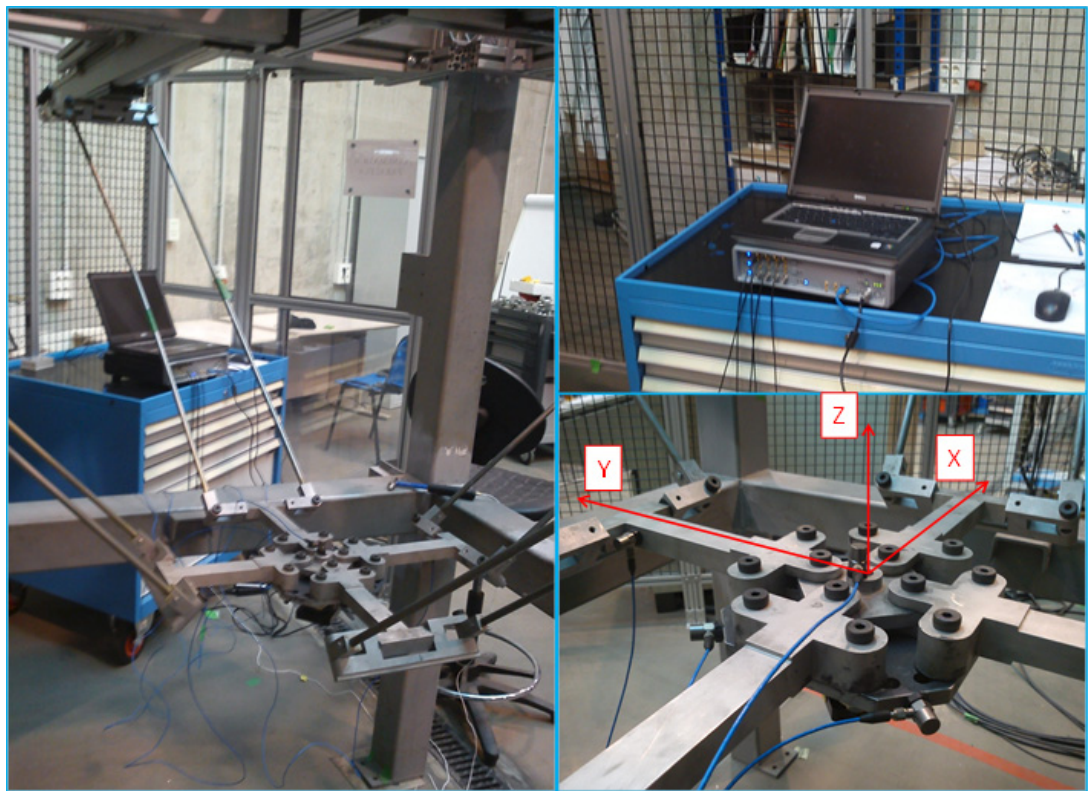

Fig. 10. Prototype and experimental setup.

In Figure 9, the values of the natural frequency are plotted for $\theta_{z}=0$ as a function of translational position. In these maps, it can be observed that the frequency varies markedly with the position of the mobile platform in the workspace. The order of the natural frequencies has been determined by the order of appearance of the peak in the centre of the work plane. Defining a point of reference is critical as natural frequencies vary by position and they can interfere. Indeed, this phenomenon of interference is what is responsible for the various deformation modes appearing in a different order at different positions.

As for the static case, the dynamic vibrational modelling has also been validated. Figure 10 is a photograph of the prototype and experimental setup for vibration measurements. This setup consisted of a $L M S S C A D A S$
Mobile multi-channel fast Fourier transform (FFT) analyser, and a set of piezoelectric transducers to record the response of the manipulator to an impulse excitation generated by a hammer. Excitation forces were applied to the prototype at several different points and in all three Cartesian directions in an attempt to excite all the modes predicted by the numerical model.

\subsection{Dynamic workspace}

Imposing dynamic constraints on some of the natural frequencies, we obtain the dynamic workspace, $W_{D}$. For example, the dynamic workspace for the first natural frequency, $f_{1}$, is plotted in Figure 11. As in the static case, 


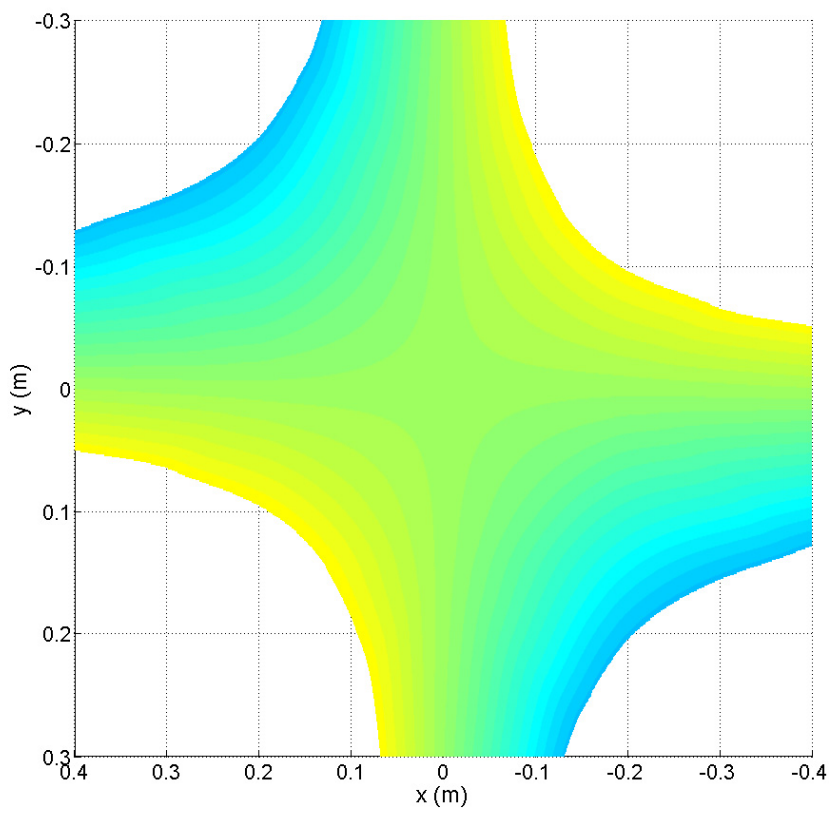

Fig. 11. Dynamic workspace, $W_{D}$.

the dynamic workspace is a subregion of the operational workspace, $W_{\text {op }}$.

$$
W_{D}=\left\{W_{\mathrm{op}} \backslash f_{i_{\min }}<f_{i}<f_{i_{\max }}\right\}
$$

\section{Results}

Finally, on the basis of the workspaces presented, we are able to identify a workspace that enables all of the kinematic, static and dynamic constraints to be fulfilled. The result is the structural workspace, $W_{S}$, that corresponds to the intersection of the aforementioned constraints, that is, the geometrical region covering the points that simultaneously meet all of the structural requirements:

$$
W_{S}=W_{K} \cap W_{D}
$$

The structural workspace for the case study is shown in Figure 12. These results can be applied directly for tool trajectory planning, meeting the requirements for minimum stiffness and a given range of natural frequencies.

\section{Conclusions}

In this paper, we have characterised the static properties of the manipulator in terms of stiffness, leading to a description of the static behaviour of the system in the operational workspace, $W_{\text {op }}$. Adding static constraints, the static workspace, $W_{K}$, has then been defined. In relation to the vibrational properties (natural frequencies and modes), we have explored the dynamic behaviour of the system using natural frequency maps, while the natural modes have also been considered; we have observed

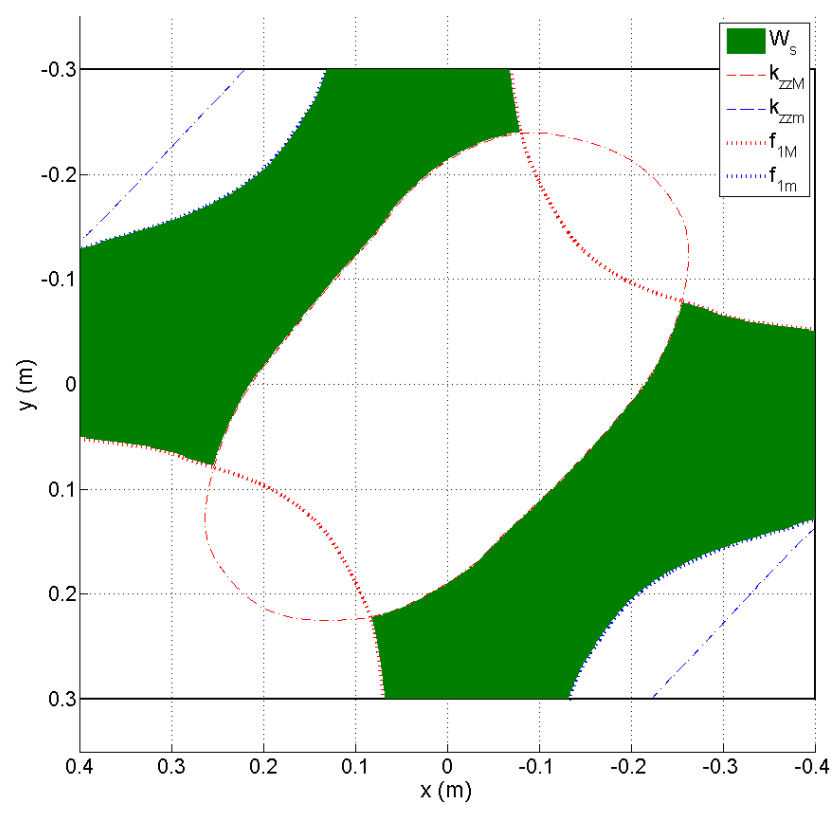

Fig. 12. Structural workspace, $W_{S}$.

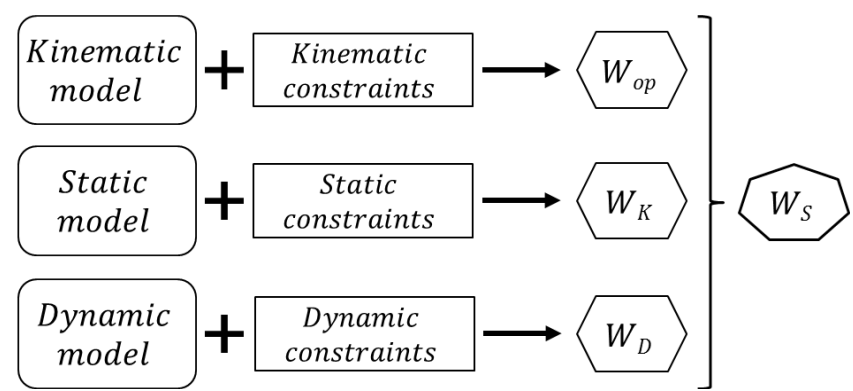

Fig. 13. Flow diagram of the characterisation method.

that bending is the dominant mode in the dynamic deformation and the greatest effect is seen at the point where the tool is to be mounted. In a similar way to the static case, the dynamic workspace, $W_{D}$, has been defined, for a specific range of natural frequencies. Finally, we have obtained the structural workspace, $W_{S}$, a subregion of the operational workspace in which the system meets the structural requirements for the application. This study, given its general nature, enables us to propose a methodology for the structural characterisation of parallel manipulators based on structural workspaces and this is summarised in Figure 13.

In particular, the structural characterisation described here makes it possible to determine values of modal parameters for a manipulator in its workspace. To assess the suitability of the manipulator for a specific machining application, these values could be fed into a model of the system behaviour for the corresponding process.

Acknowledgements. The authors of this work wish to acknowledge the financial support received from the Spanish Government through the Ministerio de Educación y Ciencia (Project DPI2011-22955) and the regional Government of the Basque 
Country through the Departamento de Educación, Universidades e Investigación (Project IT445-10).

\section{References}

[1] F. Pierrot, V. Nabat, O. Company, S. Krut, P. Poignet, IEEE Transactions on Robotics 25 (2009) 213

[2] D. Chablat, P. Wenger, F. Majou, J.P. Merlet, International Journal of Robotics Research, 2004, pp. 1-18

[3] S. Briot, V. Arakelian, S. Guegan, J. Mech. Des. 130 (2008) 122303

[4] O. Company, F. Pierrot, J. Fauroux, in Proceedings of the ICRA 2005, 2005, pp. 3232-3237

[5] S.K. Dwivedy, P. Eberhard, Mechanism and Machine Theory 41 (2006) 749

[6] Y. Wang, H. Liu, T. Huang, D.G. Chetwynd, Journal of Mechanisms and Robotics 1 (2009) 21002

[7] Y.K. Yiu, H. Cheng, Z.H. Xiong, G.F. Liu, Z.X. Li, in Robotics and Automation, 2001. Proceedings 2001 ICRA, IEEE International Conference on 4 (2001) 3766-3771

[8] G.J. Wiens, D.S. Hardage, in International Design Engineering Technical Conferences \& Computers and Information in Engineering Conference ASME, 2 (2006) $749-758$
[9] C. Pinto, J. Corral, O. Altuzarra, A. Hernández, Robotica 28 (2009) 719

[10] R. Rizk, M. Munteanu, J.C. Fauroux, G. Gogu, in 12th IFToMM World Congress, Vol. 2, 2007, pp. 1-6

[11] F. Xi, D. Zhang, C.M. Mechefske, S.Y.T. Lang, Mechanism and Machine Theory 39 (2004) 357

[12] D. Zhang, F. Xi, C.M. Mechefske, S.Y.T. Lang, Robotics and Computer-Integrated Manufacturing 20 (2004) 151

[13] J.P. Merlet, Parallel robots (Springer, 2006)

[14] Z. Li, J. Wang, X. Peng, S. Li, Z. Yin, American Journal of Nanotechnology 1 (2010) 86

[15] H. Chanal, E. Duc, P. Ray, Int. J. Machine Tools Manuf. 46 (2006) 98

[16] T. Bonnemains, H. Chanal, B.C. Bouzgarrou, P. Ray, J. Manuf. Sci. Eng. 131 (2009) 041013

[17] O. Salgado, Síntesis, análisis y diseño de manipuladores paralelos de baja movilidad, Ph.D. thesis, University of the Basque Country, 2008

[18] K. Arrouk, B.C. Bouzgarrou, G. Gogu, in 19th International Workshop on Robotics in Alpe-AdriaDanube Region, 2010, pp. 155-160 